ÉGYPTE monde arabe

\section{Égypte/Monde arabe}

$3 \mid 2000$

La censure ou comment la contourner

\title{
Agenda: Un nouvel ordre du jour pour la censure?
}

\section{(2) OpenEdition}

1 Journals

Édition électronique

URL : https://journals.openedition.org/ema/817

DOI : 10.4000/ema.817

ISSN : 2090-7273

\section{Éditeur}

CEDEJ - Centre d'études et de documentation économiques juridiques et sociales

\section{Édition imprimée}

Date de publication : 30 juin 2000

Pagination : 237-243

ISBN : 2-87027-856-X

ISSN : $1110-5097$

\section{Référence électronique}

" Agenda: Un nouvel ordre du jour pour la censure ? », Égypte/Monde arabe [En ligne], 3 | 2000, mis en ligne le 08 juillet 2008, consulté le 07 juillet 2022. URL : http://journals.openedition.org/ema/817 ;

DOI : https://doi.org/10.4000/ema.817

Ce document a été généré automatiquement le 7 juillet 2022.

Tous droits réservés 


\section{Agenda: Un nouvel ordre du jour pour la censure?}

1 Il y a quelque trente ans, le sociologue et essayiste syrien Abû cAlî Yâsîn avait stigmatisé, dans al-Thalûth al-muharram (Le Trio interdit) les trois tabous qui entravent la liberté de pensée et de création et sur lesquels bute tout discours d'émancipation dans les sociétés arabes, à savoir la politique, le sexe et la religion. La littérature représente sans doute le domaine qui a conquis le plus d'espace de liberté, mais elle se heurte de plus en plus - depuis ce que d'aucuns appellent le «retour de l'islam» - à la persécution de ceux qui, au nom de cette religion, veulent imposer leurs normes à l'ensemble des discours et des pratiques, d'une part, et à celle des autorités politiques et judiciaires, d'autre part. Un quatrième interdit est venu s'ajouter aux trois premiers, comme le fait remarquer le même sociologue dans un récent ouvrage sur la littérature de la nukta1- celui qui concerne l'obscénité (badhâ’a). «J'ai découvert, écrit-il, que la littérature éminemment populaire de la nukta ploie sous le poids d'un quatrième tabou, celui de l'obscénité, c'est-à-dire ces mots qui, comme on dit, outragent la pudeur. Alors que chacun d'entre nous entend à longueur de journée les insultes et les expressions les plus obscènes, ou les prononce lui-même, nous ne présentons aux gens qu'une culture "polie". Notre littérature contemporaine est d'autant plus polie qu'elle est généralement une littérature d'intellectuels hautains ou de médias sous contrôle. Notre littérature ancienne n'était pas ainsi, de même que notre littérature populaire est mal polie, notamment dans sa partie non consignée, cette partie authentique qui n'a pas été polie... L'obscénité est parfois nécessaire (...), elle est l'arme des faibles et des opprimés. » Le même constat a déjà été fait par Khâlid al-Qashtînî, quand il mettait au point son étude sur « l'ironie politique arabe ». Contraint de la rédiger en anglais et de la faire traduire " convenablement » en arabe pour trouver un éditeur, il dut supprimer certaines anecdotes et en corriger d'autres dans la version arabè2. Ahmad Bahâ' al-Dîn rapporte quant à lui l'entretien qu'il eut avec Sadate au lendemain des émeutes de janvier 1977, baptisées « émeutes des voleurs " par le président égyptien (intifâdat alharâmiyya), trés indigné alors par les «slogans extrêmement obscènes " criés par la «multitude »: «Vous dites souvent que le peuple d'Égypte est parmi les peuples les plus racés de la terre et qu'il a une histoire de plus de 7000 ans; mais, entre nous, n'est-il pas aussi l'un des peuples les plus "obscènes" du monde? Peut-on faire dix pas 
dans n'importe quelle rue sans entendre les gens prononcer les mots les plus obscènes ?... Je lui disais cela en plaisantant... mais je me suis heurté à un mur... » $^{3}$

Les recensions et commentaires qui suivent illustrent l'empire toujours vivant des quatre tabous déjà cités et les ravages qu'ils sont encore capables de provoquer; et ce, même s'ils sont régulièrement dénoncés dans des écrits ou des colloques, tels la contribution du critique de cinéma et ex-directeur de la censure (!), cAlî Abû Shâdî (« Le terrorisme contre la pensée et la liberté de création ») ou le colloque sur la liberté intellectuelle et académique en Égypte, organisé par le Centre de recherches arabes et coordonné par l'universitaire et militante de gauche Amîna Rashîd5.

\section{L'obscénité ou les nouvelles affaires de censure universitaire}

3 En janvier 1999, le professeur ‘Abd-al-Muncim al-Jumayĉ, du département d'histoire de l'université du Caire, a été déféré devant un conseil de discipline pour avoir inscrit au programme et avoir enseigné l'ouvrage de l'agitateur et tribun populaire cAbdallah alNadîm intitulé al-Masâmîr (Le Caire, s. 1., 1999). Écrit durant l'exil stambouliote de l'auteur au lendemain de l'échec de la révolte de cUrâbî, al-Masâmîr (Les Clous) est l'un des pamphlets les plus atroces et les plus obscènes de la prose arabe mais depuis longtemps reconnu comme un classique. Il fut rédigé contre l'homme de religion le plus proche du sultan cAbd al-Hamîd II, le libanais Abû-l-Huda al Sayyâdî, lequel vouait une haine déclarée à l'Égypte des khédives, baptisée « demeure de la débauche, de la licence et du libertinage ». L'ouvrage, bien connu des spécialistes de la littérature politique du XIX siècle, a déjà fait l'objet de recherches ou d'enseignement de la part d'historiens tel que Muhammad Fu'âd Shukrî, Muhammad Anîs ou Ra'ûf cAbbâs. Pour son enseignement, al-Jumayî a réédité l'ouvrage dont il existe un exemplaire à la bibliothèque de l'université. Mais l'un de ses collègues mal intentionné en a photocopié quelques pages qu'il a jointes à un petit rapport qu'il a rédigé et adressé au recteur de l'université, dénonçant le libertinage et les obscénités dont l'ouvrage serait plein. AlJumayî̀ a aussitôt été déféré devant une commission d'enquête et le livre retiré du programme. On dit que l'enseignant-délateur a été lui-même déféré devant un conseil de discipline par al-Jumayô en tant que vice-doyen de l'université pour avoir vendu les sujets d'examens aux étudiants...

4 En plein scandale Haydar Haydar, l'hebdomadaire al-Maydân puis le quotidien al-Akhbâr lancent ce qu'ils appellent « l'affaire du département de sociologie » de l'université de Mansûra. Ils se font l'écho des parents d'étudiants qui, après avoir vainement fait appel au doyen de la faculté des lettres puis au recteur de cette université, ont dû recourir aux journaux pour " protéger " leurs enfants et attirer l'attention des autorités sur ce qu'ils considèrent comme un outrage aux bonnes mœurs et à la pudeur. En fait, ce que dénoncent ces parents et les journalistes qui les ont défendus, c'est qu'on puisse mettre au programme de quatrième année de sociologie, un ouvrage rédigé par le professeur M. A. Ghunaym (qui est aussi vice-doyen de la faculté des lettres de Mansûra), intitulé al-Jarîma wa inhirâf alahdâth (Le crime et la délinquance juvénile), et que cet ouvrage soit publié et subventionné par l'université (Publications de l'université de Mansûra, 2000), alors qu'il est « plein d'expressions obscènes ». L'effet est immédiat puisque l'auteur est déféré devant un conseil de discipline et le livre retiré de la vente, quoique, ajoutent les parents, « les faibles d'esprit s'arrachent malheureusement cet ouvrage qui se vend au 
marché noir ». En réalité, il s'agit d'un manuel ordinaire de sociologie criminelle, mais qui contient, sous forme d'annexes, des procès verbaux d'interrogatoires menés par la police des mœurs (al-âdâb) auprès de jeunes prostituées avant de les déférer au Parquet. Ce sont les réponses de ces «jeunes délinquantes» qui contiennent nombre d'expressions "vulgaires" et de mots "crus", puisque les organes sexuels sont naturellement appelés par leurs noms. Voir écrits en toutes lettres, dans un ouvrage inscrit au programme, des mots et des expressions qui n'ont jamais accès à l'écrit et que seuls les "voyous » sont capables de prononcer, voilà ce qui a fait scandale et semblé tout à fait intolérable pour la majorité des étudiants et des parents. Comme le remarque avec tristesse ou indignation de nombreux enseignants-chercheurs, ce n'est pas seulement la liberté d'enseignement qui est remise en cause mais l'esprit même de la recherche scientifique au nom de valeurs morales et religieuses qui ont peu à voir avec la science (cf. al-Ahrâm al-arabî, 10/06/2000). Les journalistes s'en mêlent et donnent des leçons aux universitaires: "Le professeur qui a reproduit les procèsverbaux de la police des mœurs aurait dû, écrit al-Akhbâr, prendre la peine de les purger des mots vulgaires, obscènes et contraires à la pudeur publique. Aussi, conclut le journal, il faut qu'existe une instance qui puisse contrôler ce qu'enseignent les honorables professeurs à nos enfants étudiants. " Les sociologues interrogés ont fait remarquer avec justesse que le manuel en question s'adresse à des étudiants adultes qui sont censés devenir très bientôt des sociologues ou des travailleurs sociaux, et auront de ce fait à affronter des phénomènes sociaux que la morale condamne peut-être, mais qui existent bel et bien. Au cours d'une recherche sur la prostitution menée dans les prisons, « il ne faut pas s'attendre à entendre le langage de la haute littérature ou celui des soufis » dit un sociologue du CNRSC ${ }^{6}$ qui ajoute : «il y a vingt-cinq ans, nous avions commencé une recherche sur le comportement sexuel des étudiants dans le cadre d'une étude sur les changements sociaux. Nous avions minutieusement préparé un questionnaire et commencé le travail de terrain, lorsqu'un journaliste, Salâh Muntasir, a lancé une attaque contre le projet et accusé ses responsables d'être des criminels en soumettant les étudiants à de telles questions... Le chef de l'étude, Abd-al-Halîm Mahmûd, alors chef du département de sociologie, a dû recourir à Mme Gihane Sadate pour arrêter la campagne de presse, mais le projet a dû être abandonné (...). Nous assistons aujourd'hui à de nombreux phénomènes sociaux, tels que le mariage urfi`entre étudiants, la fornication, le viol et l'inceste (...). La société traverse une révolution sexuelle et il est nécessaire de l'étudier pour pouvoir la maîtriser. Le silence et le secret qui sont socialement de règle quand il s'agit de sexe, n'ont pas lieu d'être dans la recherche scientifique et sociologique. » (al-Ahrâm al-carabî, 10/06/2000, p. 71) Le doyen des sociologues égyptiens, Ahmad Abû Zayd, raconte, dans le même journal, une mésaventure similaire. Dans une recherche sur la «perception qu'ont les Égyptiens d'eux-mêmes et du monde qui les entoure ", menée entre 1987 et 1991 au sein du CNRSC, «j'ai rédigé une première partie intitulée "La perception de soi", et j'ai commencé par le problème de "la perception du corps", soit quelque 200 pages où j'ai abordé la dualité du corps et de l'âme et fait remarquer que la perception des Égyptiens surévaluait la partie supérieure du corps. Des objections ont été soulevées quand nous avons soutenu que le sexe est le langage du corps; et ce, à partir de ce qu'ont dit les gens eux-mêmes et non d'après mes propres opinions. Mais les responsables du centre [le CNRSC] ont craint les objections venant de l'extérieur et m'ont demandé de revoir le texte après avoir suspendu sa publication. Ce que j'ai refusé, par respect pour les valeurs de la recherche scientifique, d'autant plus que ce qu'on m'a demandé de 
corriger, ce sont les expressions utilisées par les gens au cours de l'enquête. (...) J'ai refusé par la suite de publier cette étude chez un autre éditeur, pour des raisons éthiques et professionnelles, car le centre avait assuré le financement et fourni l'équipe de recherche..." Ahmad Abû Zayd estime que "la peur du sexe» est due à l'environnement politique dominé par les forces conservatrices dont on craint les réactions. Pour lui, un tel sujet aurait été tout à fait ordinaire pendant les années quarante et aurait pu être publié in extenso sans soulever aucune objection; "Alors, Ismâîl Adham pouvait publier un livresintitulé Pourquoi je suis athée et M. Farîd Wagdî lui répondre par un autre livre: Pourquoi je suis croyant (...). Et si les responsables avaient lu les ouvrages des Anciens et ce qu'ont écrit les fuqahâ' sur le sexe, ils auraient autorisé la publication de l'étude. »

\section{L'athéisme revendiqué ou l'affaire Salâh al-dîn Muhsin}

Le 7 juillet 2000, la cour de sûreté de l'État a condamné l'écrivain Salâh al-dîn Muhsin à six mois de prison avec sursis, après un procès de plus de deux mois, conclusion d'une affaire de "blasphème" que la plupart des médias se sont gardés de porter à la connaissance du public et que les intellectuels, qu'ils soient laïques ou islamistes, ont ignoré avec superbe. La note qui suit est rédigée à partir des informations publiées par les rares journaux qui ont évoqué l'affaire, elles-mêmes tirées du rapport de police qui a servi à l'inculpation de l'auteur de Irticâshât tanwîriyya.

6 Salâh al-dîn Muhsin est un écrivain autodidacte âgé de 52 ans. Il a obtenu un baccalauréat commercial en 1967 et n'a pas fait d'études supérieures. Après avoir occupé les fonctions de magasinier dans un village de la province de Gîza, puis de secrétaire comptable au Centre de recherches agricoles, il émigre au Liban où il travaille dans une imprimerie, puis en Irak où il passe trois ans en tant que manœuvre. Il rentre en Égypte en 1980 et renonce à son poste de fonctionnaire afin, dit-il, de «se consacrer aux grandes causes et écrire des livres». Pour vivre, il travaille en tant qu'agent commercial dans une société de peinture. Entre 1980 et 1991, il publie, à compte d'auteur, quelques petits ouvrages littéraires (Les anecdotes de Bokassa, 1980 ; Le guide des amants, 1982; Sociologie orientale, 1984) ainsi que quelques autres qu'il juge " sans importance ».

7 À partir de 1992, il commence ce qu'il considère l'œuvre et la mission de sa vie : la critique de la religion. Après avoir lu le Coran et les livres de figh, il découvre, reconnaît-il, qu'ils contiennent des contradictions et des affirmations contraires à l'esprit du temps et à la science moderne. Il découvre aussi que la sharia est le grand obstacle qui entrave le progrès des peuples musulmans et qu'y croire et suivre ses commandements c'est se condamner à l'arriération et s'éloigner de la civilisation. Par la même occasion, il en arrive à la négation de l'existence de Dieu pour affirmer que le Coran est rédigé par le prophète Muhammad. Les idées qu'il propageait dans son

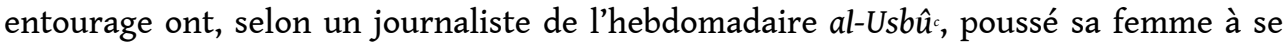
séparer de lui.

8 Le 10 mars 2000, les services de la Sûreté de l'État reçoivent une dénonciation contre un livre intitulé Irtiâshât tanwîriyya (Frissons qui éclairent) qui contiendrait des affirmations contraires à la sharia et hostiles à la religion et que son auteur, le nommé Salâh al-Dîn Muhsin, vend lui-même au prix de 5 livres égyptiennes l'exemplaire. Aussitôt, l'avocat général confie l'affaire à Ashraf 'Ashmâwî, juge d'instruction au 
parquet de la Cour de sûreté de l'État, lequel ordonne l'arrestation de Muhsin. Au cours de la perquisition, la police saisit quelques centaines d'exemplaires de ses quatre derniers ouvrages et nombre de publications périodiques que l'accusé diffuse lui-même. Seul le Centre d'aide juridique pour les droits de l'homme publie un communiqué pour protester contre son arrestation, considérée comme une violation flagrante des articles 46 et 47 de la Constitution qui garantissent la liberté d'opinion, d'expression et de croyance.

9 L'accusé étant membre de l'Union des écrivains, le Parquet a aussitôt ouvert une enquête auprès des responsables de la corporation afin de connaître les conditions de son affiliation. Fârûq Shûsha, président, a affirmé que S. Muhsin a été admis sur la base de rapports rédigés par deux écrivains reconnus : ‘Abd al-cÂl al-Hamamsî, secrétaire de l'Union et Nihâd Sharîf, spécialiste de la littérature de science-fiction. Les deux rapports ont donc été versés au dossier de l'affaire. Le premier, fondé sur la lecture de quatre ouvrages de Muhsin, aboutit à l'idée que l'auteur possède une capacité de communication avec le public, bien que ses écrits relèvent plus du prêche que de l'art romanesque; il recommande son adhésion en signe d'encouragement et non de reconnaissance. Nihâd Sharîf, quant à lui, nie à l'ouvrage qu'on lui a demandé d'examiner (Musâmarât al-samâ') son appartenance au genre de la science-fiction; il le trouve "plein de fantasmes débridés qui se transforment en une sorte de récit mythique où sont injectées les idées politiques et religieuses de l'écrivain ". Toutefois, l'auteur du rapport voit qu'il n'y a pas de mal à l'admettre comme membre de l'Union et la commission d'enregistrement a entériné cet avis.

D'après la liste donnée sur la quatrième de couverture de son avant-dernier ouvrage ( $L a$ libération de l'homme), S. Muhsin a écrit une quinzaine d'ouvrages, entre essais, recueils de nouvelles, pièces de théâtre et romans. Mais les quatre ouvrages pour lesquels il a été jugé sont :

- $1^{\circ}$ ) Musâmarât al-samâ' (Causeries avec le ciel, 1992), dialogue entre deux personnages qui commentent avec ironie certains versets du Coran, les pastichent avec dérision et se moquent du Prophète et de ses compagnons.

- $2^{\circ}$ ) cAbcâtî (Contraction du prénom 'Abd-al-cÂtî, 1998), raconte la vie d'un villageois analphabète mais qui aime la compagnie des lettrés pour parler de questions religieuses. L'auteur rapporte des dialogues où le personnage s'adresse à Dieu sans aucune marque de respect ou de vénération, se moque même de lui et de certaines de ses affirmations sur le jugement dernier, l'enfer et le paradis. L'ouvrage se conclut par un réquisitoire contre la sharia considérée comme vaine, désuète et inapplicable.

- $3^{\circ}$ ) Mudhakkirât muslim (Mémoires d'un musulman, 1998), sorte d'autobiographie où l'auteur raconte sa propre vie entre 1954 et 1998, en décrivant l'évolution de ses croyances religieuses et son attitude vis-à-vis de l'islam et de l'existence de Dieu. Il arrive à la conclusion que la religion de Muhammad est un ramassis de légendes héritées de génération en génération, et ce dans un style iconoclaste, jugé outrageant et sacrilège. Il se moque des cinq piliers de l'islam et de ceux qui les respectent, rejette le Coran et la Sunna et les tourne en dérision. L'ouvrage se termine par ce que l'auteur appelle son testament où il recommande à ses amis et à ses proches de remplacer la récitation du Coran, lors des cérémonies funéraires après sa mort, par des chansons de Fayrûz et de cAbd al-Wahhâb.

- $4^{\circ}$ ) Irticâshât tanwîriyya (Frissons qui éclairent, 1999), son dernier ouvrage et celui qui a causé son arrestation. Il y appelle à une nouvelle ère des Lumières, fondée sur le rejet des religions, plus particulièrement l'islam, décrit comme étant une mythologie exprimant 
«l'ignorance sacrée des bédouins et héritée depuis quatorze siècles ». Il incite les musulmans à ne plus croire qu'à la science moderne, car le livre qu'ils vénèrent, le Coran, est un tissu de contradictions et d'erreurs scientifiques, voire rhétoriques et grammaticales. Puis il s'attaque aux intellectuels et journalistes qui se réclament des Lumières (tanwîr) tels qu'Anîs Mansûr, Gâbir cUsfûr, Ragâ al-Naqqâsh, Sayyid al-Qinînî et Hasan Hanafî, qu'il accuse de pratiquer l'anesthésie intellectuelle et de manquer d'audace dans leur critique de la religion.

11 Commencé le 17 juin 2000 devant la cour de sûreté de l'État, le procès a peu attiré l'attention de la presse. L'auteur, qui avait refusé de renoncer à ses idées antireligieuses au cours de l'instruction, a adopté lors du procès une attitude défensive, estimant qu'on lui faisait un procès d'opinion. Ses avocats ont plaidé cette cause, affirmant qu'il n'a rien fait de plus qu'exprimer ses idées philosophiques sur la vie et l'univers et relever les contradictions qu'il y a entre les découvertes des sciences modernes et certains versets du Coran. Se prévalant de ce qu'il croyait être « l'ère de la liberté de création », S. Muhsin a rejeté les accusations de «blasphème » et "d'outrage à la religion » et a affirmé devant ses juges qu'il aurait dû être jugé par l'Union des écrivains et non pas être jeté en prison avec les voleurs et les bandits.

Pour sa part, le procureur a dénoncé les publications qui sont apparues durant la deuxième moitié du XXe siècle pour répandre, dans « la société musulmane d'Égypte ", la pornographie au nom de l'éducation sexuelle, le blasphème et l'athéisme au nom de la raison et des Lumières. Aussi a-t-il invité le ministère de la Culture et l'Union des écrivains à prendre leur responsabilité afin qu'ils barrent la voie à de prétendus « créateurs » tels que S. Muhsin.

13 La cour a par la suite rejeté la requête de la défense d'écouter les témoignages du ministre des Waqf cheikh Hamdî Zaqzûq, du secrétaire général du Haut Conseil de la culture Gâbir cUsfûr et des écrivains Sayyid al-Bahrâwî et Salwa Bakr; probablement pour ne pas transformer le jugement en débat d'idées. Le verdict, rendu après la clôture de l'affaire Haydar Haydar, est plutôt clément.

Cependant, l'affaire S. Muhsin a révélé, une fois de plus, les contradictions des intellectuels laïques. Ainsi, Salâh Fadl, professeur de littérature et défenseur ardent du roman de Haydar Haydar, Un Festin pour les algues de mer, au sein de la "commission scientifique » désignée par le ministre de la Culture a proposé à l'Union des écrivains de rayer le nom de Salâh Muhsin de la liste de ses membres... Pour lui, «il faut empêcher ceux qui n'ont aucune qualité (compétence) intellectuelle de s'attaquer aux principes sacrés de manière crue et de s'infiltrer dans le système social sans posséder la stature ou le don leur permettant de jouir d'un respect minimum auprès de leurs lecteurs (...). L'Union des écrivains, qui défend la véritable création dans le roman de Haydar Haydar est celle-là même qui condamne ces écrits faibles et inconsistants qui nuisent à la littérature et aux écrivains. De fait, ce prétendu auteur ne méritait même pas d'être jugé !» 


\section{NOTES}

1. Notion qui peut couvrir les registres de l'humour, de l'ironie, de la plaisanterie et de la blague. Pour un ouvrage récent sur la nukta, voir Abû cAlî Yâsîn, Bayân al-hadd bayn al-hazl wa-l-jidd : dirâsa fi adab al-nukta, Damas, 1996.

2. Al-Sukhriya al-siyâsiyya al-carabiyya, trad. Kamâl al-Yâzigî, Londres, Dar al-Sâqî, 1988, p. 5-7.

3. Muhâwarâtî mac al-Sâdât, Le Caire, Dâr al-Hilâl, 1987, p. 127.

4. Dans Le terrorisme contre la pensée et la liberté de création, travaux de la Seconde rencontre intellectuelle, Le Caire, Markaz al-dîrâsât wa-l-maclûmât al-qânûniyya li-huqûq al-insân, 1996.

5. Ce colloque - dont les actes sont publiés sous le titre Al-hurriya al-fikriyya wa-l-akâdimiyya fî misr (La liberté intellectuelle et académique en Égypte), Le Caire, Markaz albuhûth al-carabiyya, 2000 (édition scientifique : Amîna Rashîd) - est dédié à « l'avocat du peuple et l'avocat des libertés » A. N. al-Hilâlî.

6. Centre national de la recherche sociale et criminologique.

7. Mariage « coutumier » religieusement valable mais non enregistré juridiquement.

8. En fait, un article publié dans une revue littéraire.

\section{INDEX}

Mots-clés : censure 